\title{
Mycoplasma pneumonia with fulminant evolution into diffuse interstitial fibrosis
}

\author{
J M KAUFMAN, C A CUVELIER, AND M VAN DER STRAETEN \\ From the Departments of Medicine and Pathology, State University Hospital, Gent, Belgium
}

ABSTRACT A fatal case of interstitial pneumonia caused by Mycoplasma pneumoniae with fulminant evolution into diffuse interstitial fibrosis is reported. Treatment with tetracycline anf corticosteroids failed to arrest the progress of the disease. Fatal Mycoplasma pneumoniäe infections have been reported previously and some degree of pulmonary fibrosis has been described in a few cases but as far as could be ascertained there are no other well-document cases of diffuse interstitial fibrosis with proved Mycoplasma pneumoniae infection.

Mycoplasma pneumoniae is a well-documented and recognised cause of pneumonia, although in most cases infection remains subclinical or limited to upper respiratory tract involvement. ${ }^{1-4}$ Clinical and radiographic features are inconstant and do not allow for differentiation from pneumonias caused by other micro-organisms. ${ }^{56}$ Mycoplasma pneumoniae usually has a rather benign, selflimiting course. However, besides important extrapulmonary complications such as haemolytic anaemia, ${ }^{78}$ myopericarditis, ${ }^{8-10}$ and neurological manifestations, ${ }^{34}$ severe and even fatal respiratory disease from Mycoplasma pneumoniae has been reported. The pulmonary complications include unilateral and bilateral massive pneumonia, ${ }^{311} 12$ large unilateral and bilateral pleural effusions, ${ }^{3} 1113$ lung abscess, ${ }^{3}{ }^{12}$ Swyer-James syndrome, ${ }^{14}$ and extensive interstitial pneumonia with severe hypoxaemia. ${ }^{15-17}$ Although exceptional, persistence of respiratory symptoms after Mycoplasma pneumoniae infection has been reported, ${ }^{18}$ and on a few occasions some degree of fibrosis has been demonstrated at necropsy ${ }^{17-19}$ but this condition has not yet been well documented.

\section{Case report}

Sixteen days postpartum, a previously healthy 31-year-old woman complained of rhinitis and general malaise followed the next day by chills, fatigue, and non-productive cough. On the third night she woke up with mild dyspnoea which

Address for reprint requests: Dr JM Kaufman, Department of Medicine, State University Hospital, Gent, Belgium. became more pronounced during the morning? She also complained of vertigo and anorexia, an vomited once. The next morning she becanke severely dyspnoeic and was admitted to a loç hospital. Arterial blood gas analysis revealed carbon dioxide tension $\left(\mathrm{PCO}_{2}\right)$ of $35 \mathrm{mmHg}$ and oxygen tension $\left(\mathrm{Po}_{2}\right)$ of $75 \mathrm{mmHg}$ while breathing 4 litres of oxygen per minute. The chest radig graph showed patchy lobular shadows involving both lower zones (fig 1). The patient was trans? ferred to the intensive care unit of the Universit Hospital, with tachypnoea ( 36 to $40 / \mathrm{min}$ ), a ten⿳亠丷厂 perature of $37.5^{\circ} \mathrm{C}$, and slightly diminished breat

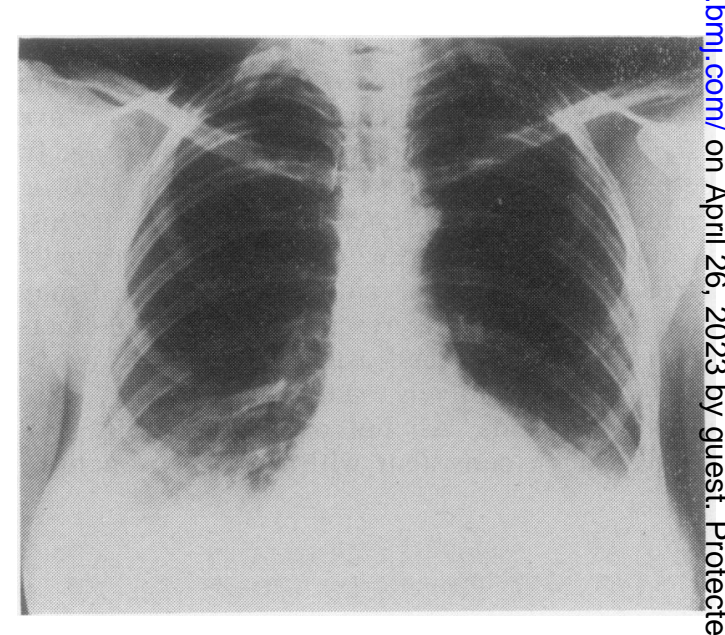

Fig 1 Chest radiograph $(P A)$ on admission to hospital shows patchy lobular infiltration involving both lower zones. 


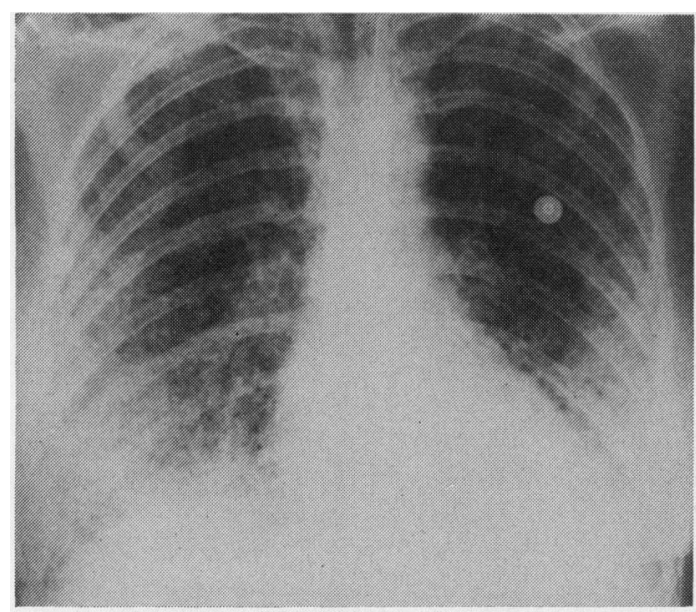

Fig 2 Chest radiograph (PA) on the fifteenth day in hospital shows diffuse mottling of both lungs.

sounds at the right base. Arterial blood gases on ambient air revealed a $\mathbf{P C O}_{2}$ of $38 \mathrm{mmHg}$ and a $\mathrm{Po}_{2}$ of $66 \mathrm{mmHg}$. The erythrocyte sedimentation rate was $100 \mathrm{~mm} / \mathrm{h}$, white cell counts $6800 / \mathrm{mm}^{3}$ with $70 \%$ neutrophils. Treatment was started with doxycycline intravenously, $200 \mathrm{mg}$ daily, and one dose of $25 \mathrm{mg}$ prednisolone.

The next day the patient seemed to improve; chest radiograph was unchanged. During the next two days, she had only mild dyspnoea but her temperature rose to $39^{\circ} \mathrm{C}$ and radiographs showed slightly increased infiltration of both bases.

A technetium perfusion lung scan showed an area of diminished perfusion in the left paracardiac area, and on the fourth day in hospital, in addition to doxycycline, heparin was started in a dose of 37500 units/day by continuous infusion. On the fifth day the patient's condition deteriorated quickly, with extreme dyspnoea and severe respiratory distress. Arterial blood gas analysis breathing 10 litres/min with an oxygen mask, showed pH 7.58, $\mathrm{PCO}_{2} 29.5 \mathrm{mmHg}$, and $\mathrm{PO}_{2}$ $34.5 \mathrm{mmHg}$. The patient became apnoeic and was immediately intubated and ventilated. Doxycycline and heparin were continued, and in addition the patient received high doses of betamethasone. For two days there was a progressive improvement with satisfactory blood gas values on PEEP $10 \mathrm{~cm}$ $\mathrm{H}_{2} \mathrm{O}$ and $50 \%$ inspired oxygen initially, reducing to $25 \%$ later on. The chest radiograph showed progressive changes, with some early reticular infiltrates in the upper zones. The white cell was between 15000 and $20000 / \mathrm{mm}^{3}$. On the tenth day in hospital the situation deteriorated again progressively. The patient went into gradually increasing alveolo-capillary block. Successive chest films showed increasing interstitial infiltrates all over the lungs with massive, patchy, confluent shadows in the lower parts and more reticular shadows in the upper parts with a picture suggesting advanced interstitial pneumonitis (fig 2).

Sputa were purulent, but frequent cultures for aerobic and anaerobic bacteria, mycobacteria, and fungi were all negative. The patient died on the seventeenth day in hospital. At that time the blood gas values ranged around $90 \mathrm{mmHg} \mathrm{PCO}_{2}$ and $60 \mathrm{mmHg} \mathrm{Po}_{2}$ despite ventilation. In one week, from the fourth to the tenth hospital day, the titre of complement fixing antibody for Mycoplasma pneumoniae rose from less than $1 / 4$ to $1 / 128$. No cold agglutinin titre could be demonstrated. Further studies on paired sera were negative for Coxsackie 1-6, Varicella, influenza A-B, para-influenza 1-3, adenovirus, psittacosis, ornithosis, and respiratory syncitial virus.

\section{NECROPSY}

The left lung weighed $800 \mathrm{~g}$ and the right lung $1100 \mathrm{~g}$. They both had a similar external aspect with moderate reticular anthracosis and a purplegrey to reddish appearance. The surface was nodular with fibrous pleura thickening. The lung felt firm and the cut surface was reddish-grey and white, intersected by round microcysts and white nodules. No purulent material could be expressed.

On microscopy similar changes were found in all the lobes. The sections made of formalin-fixed and paraffin-embedded lung tissue were stained with haematoxylin-eosin, PAS, Masson's trichrome, and reticulin. They showed a diffuse interstitial fibrosis (fig 3). The bronchioles were grouped in nodules, dilated and covered with a metaplastic stratified, flattened or squamous epithelium. Some showed cystic dilatation and contained mucus, occasional giant cells, histocytes, polymorph leucocytes, and monocytes.

Severe fibrosis was seen in the thickened interalveolar septa and around the bronchioles. The fibrous tissue consisted of young fusiform fibroblasts which formed collagen. Inside the fibrous tissue it was possible to distinguish small haemorrhages, oedema fluid, and the alveolar capillaries which were increased in size and number and surrounded by a cellular infiltrate. Within the fibrotic interstitial tissue the presence of monocytes and histocytes was observed. The alveolar walls were covered by cuboidal cells with abundant, foamy cytoplasm with round vesicular 


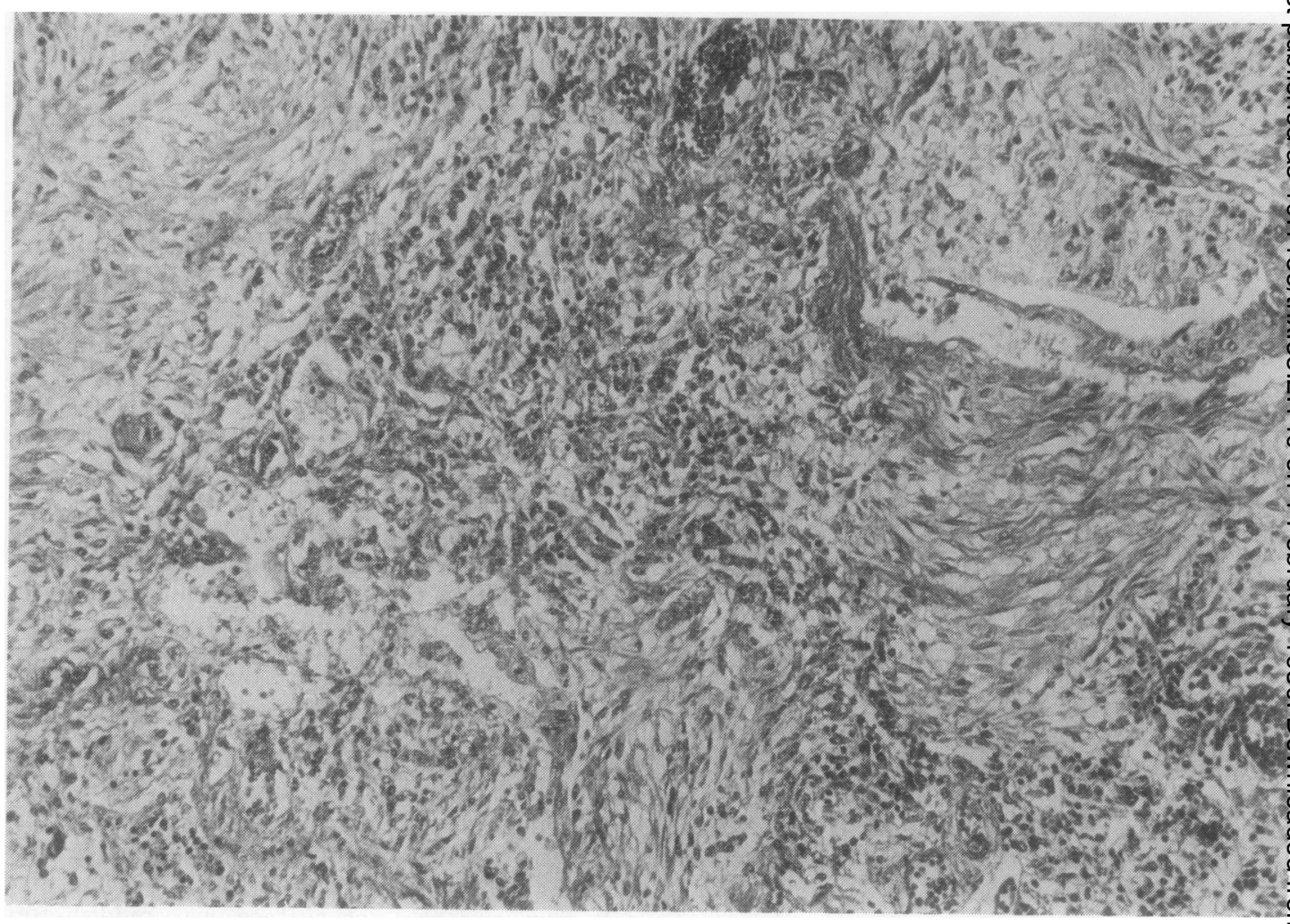

Fig 3 Severe interstitial fibrosis with fibroblast proliferation and monocellular infiltrate. Masson's trichrome stain, original magnification $\times 140$.

nuclei. Some had desquamated into the lumen. The alveoli were filled with a homogenous exudate, haemosiderin laden macrophages, polymorph leucocytes, erythrocytes, and giant cells (fig 4). Fibrin was seen only occasionally. The pleura was thickened by bundles of collagen fibres.

\section{Discussion}

Mycoplasma pneumoniae has been mentioned as a possible cause of interstitial fibrosis ${ }^{20-22}$ but only in a few cases has some degree of fibrosis been reported.17-19 There are no well-documented cases in which evolution of a Mycoplasma pneumoniae pneumonia into diffuse interstitial fibrosis has been described. The mechanisms leading to the pathological changes in diffuse interstitial fibrosis are well known but not specific. The alterations can be the result either of direct interstitial involvement or, as in this case, of a primary intraalveolar process. ${ }^{2023-27}$ The pneumocytes are desquamated into the alveolar lumen. This is followed by exudation and incorporation of the exu- date into the alveolar walls. Afterwards, fibro blast proliferation starts between the sixth and ninth day and gives rise to more organised an mature fibrous tissue. The alveolar lining eptthelium is changed into type 2 pneumocytes an the bronchiolar lining epithelium grows into the respiratory bronchiole which causes squamous metaplasia.

Numerous different agents are known to cause diffuse interstitial fibrosis. It may follow radiations therapy, oxygen toxicity, anti-neoplastic drugs, paraquat poisoning, inhalation of organic of mineral dust and toxic fumes. It is also seen as a terminal phase of sarcoidosis, collagen disease miscellaneous occupational diseases and other interstitial pneumonias. Infectious diseases have also been implicated including bacterial, viraf, fungal and parasitic infections. In our case, there was a significant rise in $M$ pneumoniae comple ment fixation titre from $1 / 4$ to $1 / 128$ and mos of the other possible aetiological agents could easily be excluded.

Oxygen toxicity may have been a factor in oum 


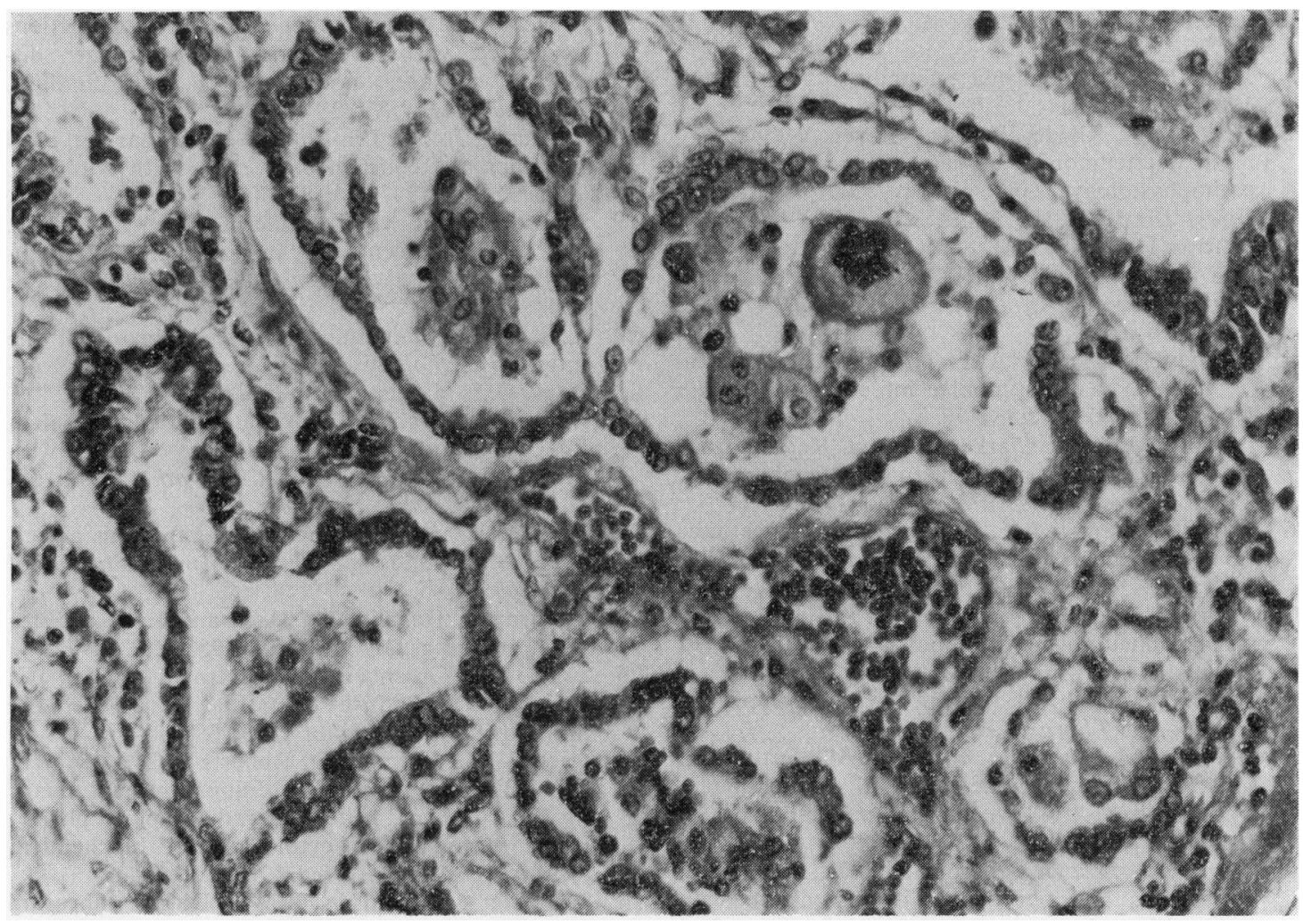

Fig 4 Alveoli covered by cuboidal pneumocytes. Thealveolar lumen is filled with macrophages, polymorph leucocytes, erythrocytes, and giant cells. Masson's trichrome stain, original magnification $\times 360$.

case, though this seems very unlikely. Indeed, our patient had been ventilated for less than 24 hours with a gas mixture containing high oxygen concentrations (maximal concentration of $66 \%$ oxygen) and at that time there was already diffuse radiological involvement of both lungs. The pathological findings in this patient could only have been provoked by prolonged administration of high oxygen concentrations. ${ }^{28-30}$

Although not well documented previously, the association of pulmonary fibrosis with Mycoplasma pneumoniae infection is not surprising, and intermediate stages such as desquamatic interstitial pneumonia have indeed been described before. ${ }^{17-19} 31$

Some authors suggest that cell-mediated immune mechanisms could be involved in severe cases of mycoplasma pneumonia. ${ }^{815}$ It is, however, not necessary to postulate an immunological mechanism to explain the pathological changes observed in our patient, by analogy with oxygen and paraquat poisoning which can result in the same histological picture without an immunological reaction. Intensive steroid therapy was not able to influence the course but administration was started only on the eighth day of illness. The lack of response to adequate therapy with high doses of tetracycline in this case is an interesting but not unique feature: resistance of Mycoplasma pneumoniae infections to tetracycline has been reported previously, and it is known that medication is effective mainly when administered early in the course of the disease. ${ }^{12732}$

High doses of corticosteroids should probably be considered in those cases of severe Mycoplasma pneumoniae pneumonia that do not improve after the fifth day from clinical onset. If started early enough steroid therapy could possibly influence fibroblast proliferation and prevent evolution into chronic or even fatal pulmonary fibrosis .

We thank Professor $\mathbf{H}$ Spencer for giving us his valued opinion on the pathological findings in this patient. 


\section{References}

1 Jones MC. Mycoplasma pneumonia. Practitioner 1969; 203:751-9.

2 Denny FW, Clyde WA Jr, Glezen WP. Mycoplasma pneumoniae disease: clinical spectrum, pathophysiology, epidemiology, and control. $J$ Infect Dis 1971; 123:74-92.

3 Murray HW, Masur H, Senterfit LB, Roberts RB. The protean manifestations of Mycoplasma pneumoniae infection in adults. Am J Med 1975; 58: 229-42.

4 Levine DP, Lerner AM. The clinical spectrum of Mycoplasma pneumoniae infections. Med Clin North Am 1978; 62:961-78.

5 Foy HM, Loop J, Clarke ER et al. Radiographic study of Mycoplasma pneumoniae pneumonia. Am Rev Respir Dis 1973; 108:469-74.

6 Brolin I, Wernstedt L. Radiographic appearance of mycoplasmal pneumonia. Scand J Respir Dis 1978; 59:179-89.

7 Maisel JC, Babbitt LH, John TJ. Fatal Mycoplasma pneumoniae infection with isolation of organisms from lung. JAMA 1967; 202:287-90.

8 Holt S, Ryan WF, Epstein EJ. Severe Mycoplasma pneumonia. Thorax 1977; 32:112-15.

9 De Vos M, Van der Straeten M, Druyts E. Myocarditis and severe bilateral bronchopneumonia caused by Mycoplasma pneumoniae. Infection 1976; 4:60-1.

10 Sands MJ, Satz JE, Turner WE Jr, Soloff LA. Pericarditis and perimyocarditis associated with active Mycoplasma pneumoniae infection. $A n n$ Intern Med 1977; 86:544-8.

11 Gump DW, Hawley HB. Severe Mycoplasma pneumoniae pneumonia. Respiration 1976; 33: 475-86.

12 De Vos M, Van der Straeten M, Druyts E. Mycoplasma pneumoniae as a cause of pneumonia, lung abscess and pleural effusion: a case report. Infection 1976; 4:58-59.

13 Chester A, Kane J, Garagusi V. Mycoplasma pneumonia with bilateral pleural effusions. $\mathrm{Am}$ Rev Respir Dis 1975; 112:451-6.

14 Stokes D, Sigler A, Khouri NF, Talamo RC. Unilateral hyperlucent lung (Swyer-James syndrome) after severe Mycoplasma pneumoniae infection. Am Rev Respir Dis 1978; 117:145-52.

15 Noriega ER, Simberkoff MS, Gilroy FJ, Rahal JJ. Life-threatening Mycoplasma pneumoniae pneumonia. JAMA 1974; 229:1471-2.

16 Nastro JA, Littner MR, Tashkin DP, Cassan SM. Diffuse, pulmonary interstitial infiltrate and mycoplasmal pneumonia. Am Rev Respir Dis 1974; 110:659-62.
17 Meyers BR, Hirschman SZ. Fatal infections associated with Mycoplasma pneumoniae: diso cussion of three cases with necropsy findings Mt Sinai J Med NY 1972; 39:258-64.

18 Parker F, Jolliffe LS, Finland M. Primary atypicaج pneumonia. Report of eight cases with autopsiesक Arch Pathol 1947; 44:581-608.

19 Benisch BM, Fayemi A, Gerber MA, Axelrod J:Mycoplasmal pneumonia in a patient with rheu $\overrightarrow{z u}$ matic heart disease. Am J Clin Pathol 1972; 582 343-8.

20 Spencer $H$. Idiopathic interstitial fibrosis of the lung. In Pathology of the lung, vol 2. Third eder ition. Oxford: Peragmon Press, 1977:728-41. N

21 Luna MA, Bedrossian CWM, Lichtiger B, Salem $\overrightarrow{\vec{D}}$ PA. Interstitial pneumonitis associated with bleomycin therapy. Am J Clin Pathol 1972; 58: 501-10.

22 Weisenburger DD. Interstitial pneumonitis associ ated with azathioprine therapy. Am J Clin Patho 1978; 69:181-5.

23 Pokorny C, Hellwig CA. Diffuse interstitial fibro sis of the lungs. Arch Pathol 1955; 59:382-7.

24 Fraire AE, Greenberg SD, O’Neal RM, Weg JGo Jenkins DE. Diffuse interstitial fibrosis of the lung. Am J Clin Pathol 1973; 59:636-47.

25 Crystal RG, Fulmer JD, Roberts WC, Moss ML Line BR, Reynolds HY. Idiopathic pulmonar ${ }_{5}^{5}$ fibrosis. Clinical, histologic radiographic, physio® logic, scintigraphic, cytologic and biochemica aspects. Ann Intern Med 1976; 85:769-88.

26 Scadding JG, Hinson KFW. Diffuse fibrosing alveolitis (diffuse interstitial fibrosis of the lungs)? Thorax 1967; 22:291-304.

27 Hinson KFW. Diffuse pulmonary fibrosis. Hum Pathol 1970; 1:275-88.

28 Bonikos DS, Bensch KG, Northway WH. Pro gressive irreversible pulmonary damage caused by chronic exposure to $100 \%$ oxygen. $A m$ b Pathol 1975; 78:9A-10A.

29 Sevitt S. Diffuse and focal oxygen pneumonitis. A preliminary report on the threshold of pulmonarys oxygen toxicity in man. J Clin Pathol 1974; 27. 21-30.

30 Simpson DL, Goodman M, Spector SL, Pett TL. Long-term follow-up and bronchial reactivity testing in survivors of the adult respiratory dis 0 tress syndrome. Am Rev Respir Dis 1978; 117? 449-54.

31 Zinserling A. Peculiarities of lesions in viral and mycoplasma infections of the respiratory tract $\sigma$ Virchows Archiv (Pathol Anat) 1972; 356:259-73.<

32 Jones GR, Borthwick RC. Mycoplasma pneu元 monia resistant to oxytetracyeline: two case re ports. Br J Dis Chest 1973; 67:119-22. 